\title{
Ecosystem services of coastal sand dunes saw from the aspect of Sake breweries in Chiba Prefecture, Japan: A comparison of coastal and inland areas
}

\author{
Korehisa Kaneko $^{1^{*}}$, Keiko Oshida ${ }^{2}$, Hajime Matsushima ${ }^{3}$ \\ ${ }^{1}$ Hokuso Creature Association, Tokyo, Japan; ${ }^{*}$ Corresponding Author: k_kaneko@hotmail.com \\ ${ }^{2}$ Department of Transportation, Engineering and Socio-Technology, College of Science and Technology, Nihon University, Chiba. \\ Japan \\ ${ }^{3}$ Research Faculty of Agriculture, Hokkaido University, Sapporo, Japan
}

Received 14 October 2012; revised 15 November 2012; accepted 1 December 2012

\section{ABSTRACT}

In this report, we reviewed the differences in the ecosystem services of coastal and inland areas surrounding 39 Sake breweries in Chiba Prefecture by investigating environmental variables (e.g., location, altitude, soil, and hardness of preparation water). The Sake breweries were located in three distinct environments: the coastal vicinity, the river plains region, and the plateaul mountainous region. The hardness of the preparation water and the soil types in the coastal vicinity were compared with those of the river plains and the plateau/mountainous region. Strong hard and hard water sources were observed in $70 \%$ or more of the breweries in the coastal vicinity, and sand dune regosol, coarse particle brown lowland soil, and coarse particle grey soil were more prevalent along the coast than inland. Most of the Sake brewery wells in the coastal vicinity were approximately $5-10 \mathrm{~m}$ underground, and there were no great differences in the number of Sake breweries in each well depth class in the river plains and the plateau/mountains region. We analysed environmental factors (distance from the sea, soil type, water hardness and preparation water collection depth) using a principal component analysis. This analysis revealed the existence of three main environments: the coastal vicinity, the river plains and the plateau/mountainous region. We conclude that the decrease in altitude between the inland Sake breweries and those along the coast is accompanied by a corresponding increase in the hardness of preparation water (from soft water to strong hard or hard water) and shifts in soil composition from gley soil, grey lowland soil, brown forest soil, and andosol to sand dune regosol, coarse particle brown lowland soil, and coarse particle grey soil.

Keywords: Ecosystem Service; Sake Brewery; Freshwater; Freshwater Layer; Soil; Hardness

\section{INTRODUCTION}

A coastal sand dune is a buffer zone between the sea and land that forms a unique ecosystem. [1] reported that the freshwater layer, which derives from the rainfall that infiltrates sands to a depth of approximately $10 \mathrm{~m}$, is used as a domestic supply of drinking and fire-fighting water. Sake breweries in the coastal vicinity are frequently located near harbours and may be influenced by river traffic. In addition, the underground freshwater layer supplies abundant mineral water, which is used for making rice malt and yeast in the Sake brewing process [2]. Japanese Sake is habitually drunk by many people and is a favourite beverage in the region. Because the methods for brewing Japanese Sake are facilitated by the climate and other natural features of the region (e.g., geographical features, soil, water quality), Sake breweries are recipients of ecosystem services.

Coastal sand dunes are formed by the repeated accumulation and invasion of river sediment [3] and cliff erosion [4]. Because the groundwater level in the hinterland is higher than the average sea level, and the freshwater that flows from the land to the sea contributes to the coastal sand dune aquifer [5], which is greatly influenced by the land area. Despite these issues, there has been no review of Sake breweries from the perspective of ecosystem services.

In this report, we review the differences in the ecosystem services of coastal and inland areas that serve Sake breweries by investigating environmental variables (e.g., location, altitude, soil, and water hardness). Our study site was the Chiba Prefecture, which contains both inland 
and coastal areas.

\section{Materials and methods}

\subsection{Study Site}

Study site consisted of 39 Sake breweries in Chiba Prefecture (including Sake breweries where home-brewing has not been conducted for several decades).

We were hearing in each Sake breweries as for the collecting place of preparation water (well, spring water, tap water) and hardness of preparation water (soft water, moderately hard water, hard water, strong hard water).

The altitude was determined using Google Earth, and the soil type was investigated using a Chiba Prefecture soil map. The environment surrounding the Sake breweries was divided into the coastal vicinity, the river plains and the plateau/mountainous region. The coastal vicinity is assumed to be a smooth geographical feature of approximately $5 \mathrm{~km}$ in length. The former natural coast was located inland of the current coastline. The river plains are characterised by smooth geographical features and low altitudes, particularly around large rivers (e.g., class A rivers). Conversely, the plateau/mountainous region boasts comparatively high altitudes (Figure 1). Figure 1 also shows the locations of the Sake breweries, some of which are located in close proximity to each other.

Ecosystem services include a "supply service", which refers to the spring water that is indispensable for the residents living near marine coastal waters. The "adjustment service" is represented by the freshwater layer under the groundwater of the coastal sand dune. Finally, the "cultural service" of the Sake breweries has supported the traditional culture in the region for several hundred years.

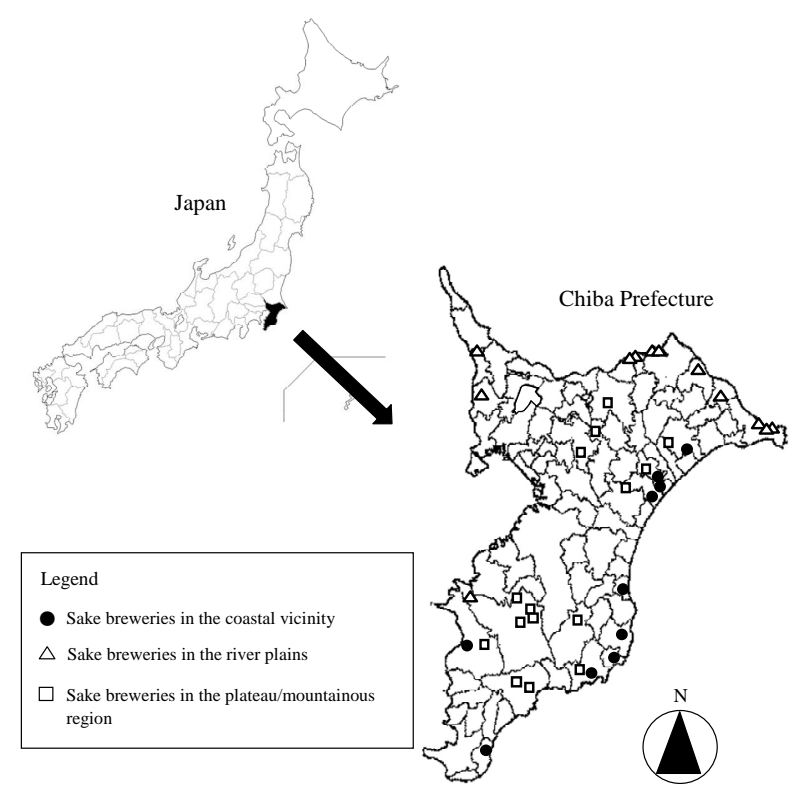

Figure 1. Study site.

\subsection{Sampling and Identification Methods}

We analysed the aforementioned environmental factors (distance from the sea, hardness of the preparation water, altitude and soil type) using principal component analysis (Excel multivariate analysis). For the water hardness analysis, soft water, moderately hard water and hard water were expressed numerically as 2, 6, and 10, which are the mean values of hardness (German hardness) used as standards by the World Health Organization. The soil type was expressed numerically as rocks 1), sand dune regosol 2), andosol 3), brown forest soil 4), redyellow soil 5), coarse particle brown lowland soil 6), grey lowland soil 7), fine particle gley soil 8), coarse particle gley soil 9), and gley soil 10).

\section{Results}

\subsection{The Environment of Sake Breweries}

There were 10 Sake breweries in the coastal vicinity, 13 along river plains, and 16 in the plateau/mountainous region. Twenty-six Sake breweries used well water as their source of preparation water (Figure 2).

The average altitudes of the coastal vicinity and the river plains were $10 \mathrm{~m}$ or less, whereas the altitude of the plateau/mountainous region was approximately $40 \mathrm{~m}$. The vertical interval was observed and compared in both the seashore environment and inland areas [2]. More specifically, according to the environmental map, which details the altitude, soil type, and hardness and collection depth of the preparation water of the various Sake breweries (Figure 3), areas falling within the 0 - $10 \mathrm{~m}$ altitude range were generally located in the coastal vicinity and river plains, whereas altitudes in the 20 - $50 \mathrm{~m}, 50$ - $100 \mathrm{~m}$, and $100 \mathrm{~m}$ or higher ranges were confined to the plateau/ mountainous region. The coastal vicinity displayed both strong hard and hard water sources, while strong hard and moderately hard water sources were detected in the river plains, and soft water was common in the plateau/ mountainous region. Regarding soil types, sand dune regosol was primarily found in the coastal vicinity, whereas

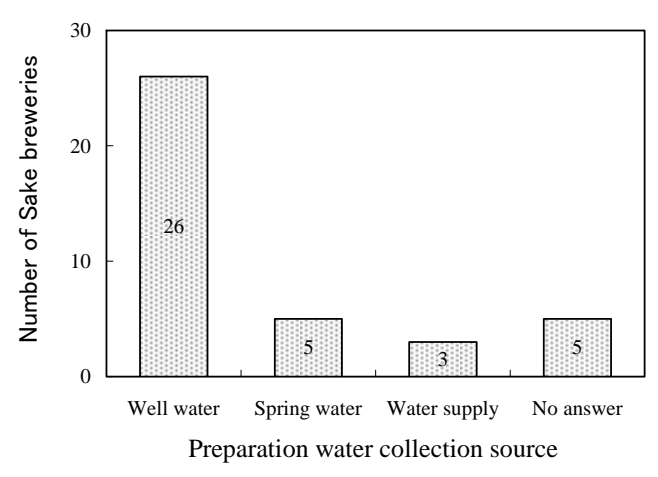

Figure 2. Distribution of preparation water collecting sources among the Sake breweries. 
the river plains featured coarse particle gley soil and gley soil, and grey lowland soil, brown forest soil and andosol were mostly confined to the plateau/mountainous area.

\subsection{The Hardness of the Preparation Water for Each Type of Environment}

We used data from the environmental map (Figure 3) to determine the relative compositions of the soil as well as the hardness and collection depth of the preparation water for each type of environment.

Strong hard and hard water comprised $70 \%$ or more of the water sources in the coastal vicinity, whereas soft water was more common in the river plains and the plateau/mountainous region (Figure 4).
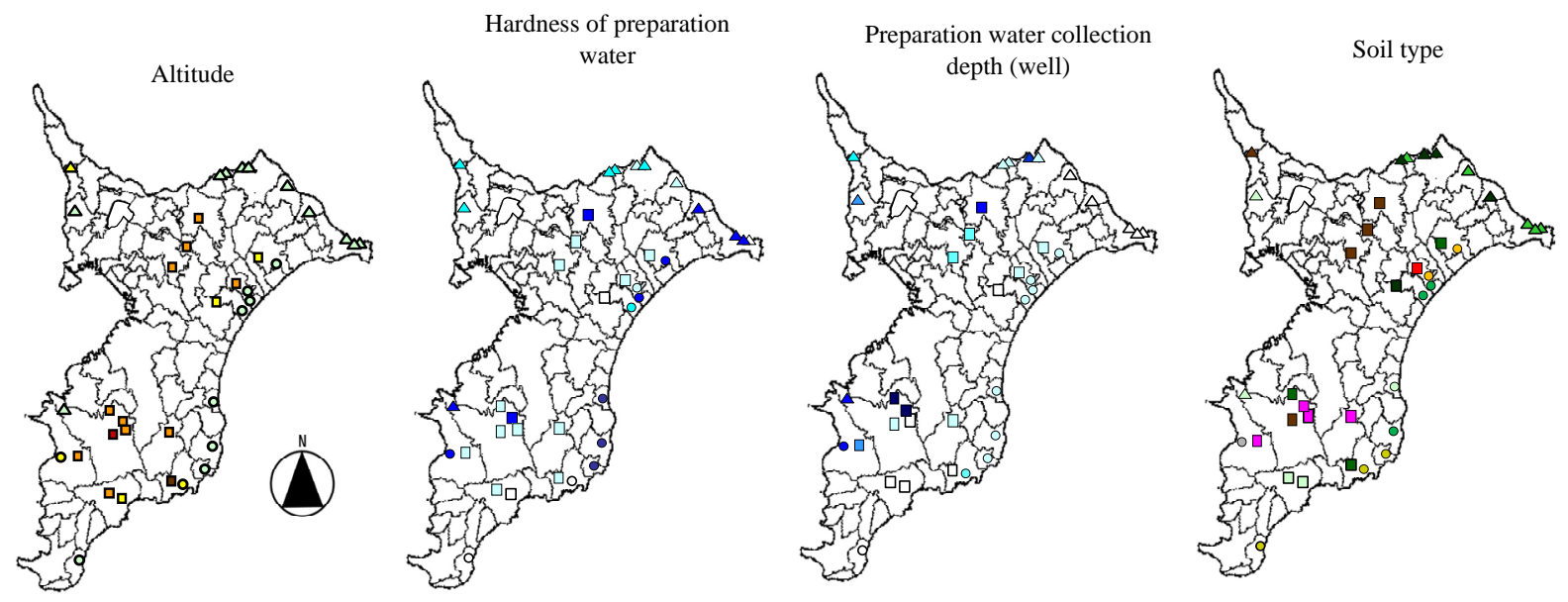

\footnotetext{
Legend

【 Each environment 】

Sake breweries in the coastal vicinity

$\triangle$ Sake breweries in the river plains

$\square$ Sake breweries in the plateau/mountainous region
}

$$
\begin{aligned}
& \text { 【 Altitude 】 } \\
& \square \text { 0-10m } \\
& \square 10-20 \mathrm{~m} \\
& \square 20-50 \mathrm{~m} \\
& \square 50-100 \mathrm{~m} \\
& \square 100 \mathrm{~m}-
\end{aligned}
$$
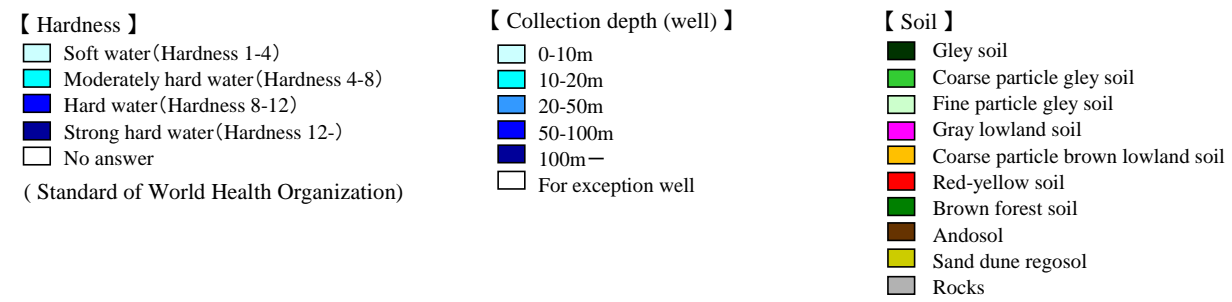

Figure 3. Environmental maps of the various Sake breweries.

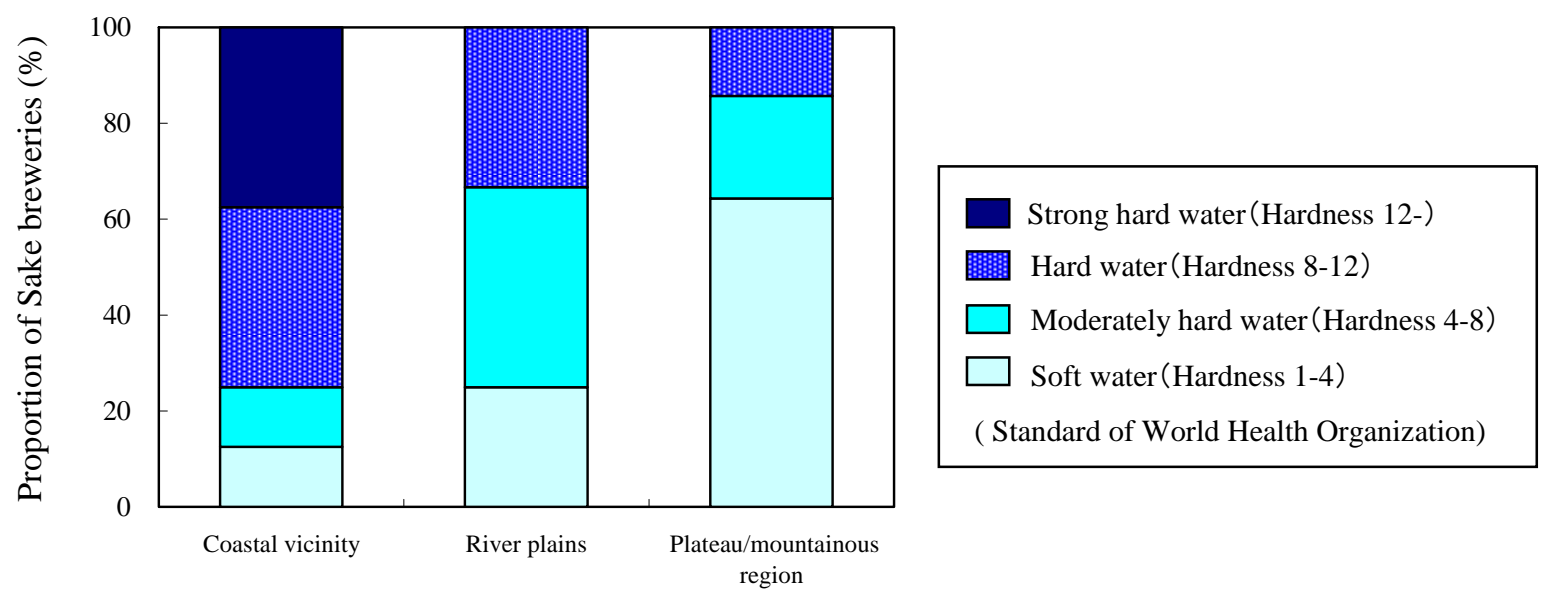

Figure 4. Composition of the preparation water hardness classes of the Sake breweries in each environment. 


\subsection{The Relative Composition of the Soil for Each Type of Environment}

In the coastal vicinity, the soil that drains into the sand dune and the low ground between the sand dunes of the marine sandy alluvial plain was mostly composed of sand dune regosol, coarse particle brown lowland soil, and coarse particle grey soil. In contrast, grainy gley soil was comparatively dominant in the river plains, and andosol, brown forest soil, grey lowland soil, and gley soil were present in the plateau/mountainous region (Figure 5).

\subsection{The Collection Depth of the Preparation Water for Each Type of Environment}

Most Sake breweries in the coastal vicinity extended approximately 5 - $10 \mathrm{~m}$ underground. There were no large differences in the number of Sake breweries in each well depth class in the river plains and the plateau/mountainous region (Figure 6).

\subsection{The Difference for Each Type of Environment}

According to the principal component analysis, if the same environments of the Sake breweries in the coastal vicinity and the river plains were excluded, three environmental types of the coastal vicinity, the river plains and the plateau/mountainous region were distinguishable (Figure 7).

\section{Discussion}

In the coastal area, strong hard and hard water comprised $70 \%$ or more of the water sources. The ground- water under sand dunes is contained by nutrients originating from the remains of seaweed and animals deposited onto the dunes and from the salt spray carried by the wind. Nutrient concentrations are also high where sand dune vegetation is abundant [6]. On the beach ridge of the Kujuukyri marine sandy alluvial plain in Chiba Prefecture, new dunes form on the upper parts of old dunes, creating a stratum demarcated by layers of humus and shells [7].

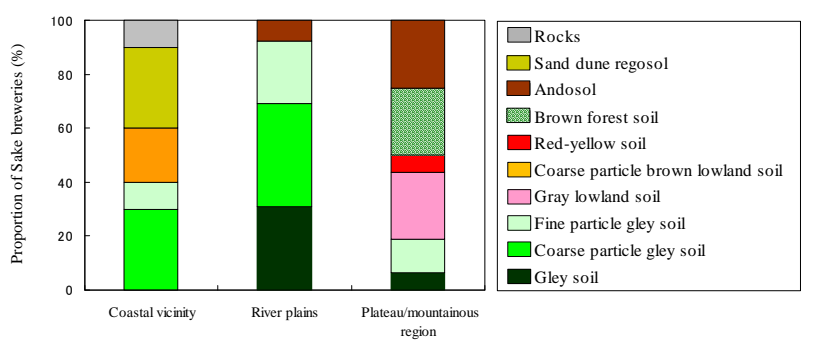

Figure 5. Soil type compositions of the Sake breweries in each environment.

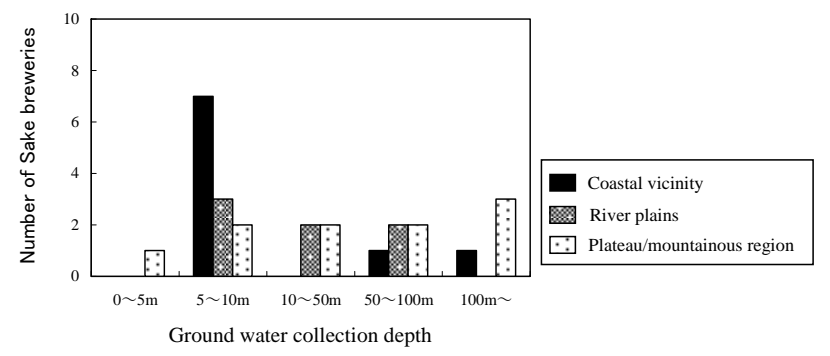

Figure 6. Groundwater collection depth at the Sake breweries in each environment.

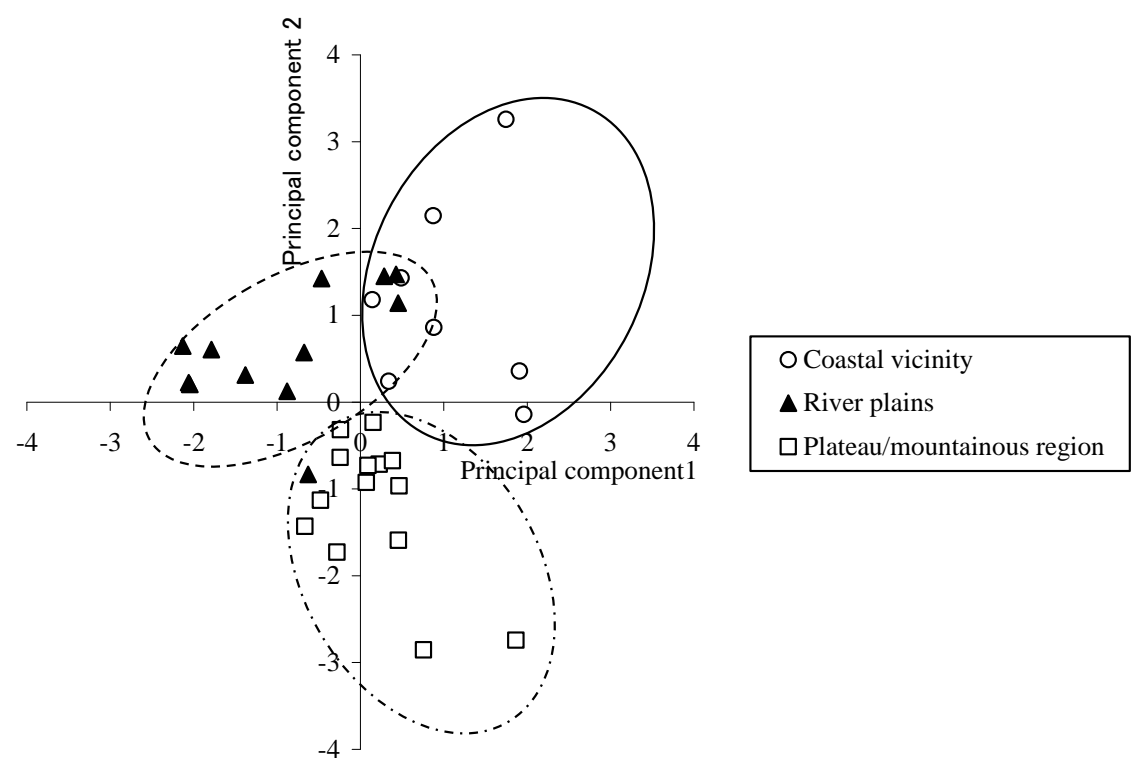

Figure 7. Environmental divisions of the Sake breweries using principal component analysis. 
In the marine sandy alluvial soil in Chiba Prefecture, potassium, phosphoric acid, calcium, and magnesium concentrations were high. The calcium concentration of the underground water that flows through the aquifer containing the shell layer has been influenced by the dissolution of splintered shells [8,9]. These nutrients actively promote the proliferation of Aspergillus oryzae and yeast, which are the predominant micro-organisms used in Sake brewing [10]. Conversely, judging from the isotope ratios, the coastal spring water in the vicinity of Mochimune in Shizuoka Prefecture originates from inland waters [11].

We suggest that the hardness of the preparation water used in coastal Sake breweries can be attributed to the underground water originating from rain that filtered through the sand stratum and the marine sandy alluvial plain layer. The latter contains high concentrations of minerals such as calcium and magnesium.

Many coastal Sake breweries use underground water from wells that are approximately 5 - $10 \mathrm{~m}$ deep. We considered this underground water to derive from the freshwater layer formed in the coastal sand dune.

The coastal areas contain a great deal of sand dune regosol, distributed on the coastal sandy ground where the drain function is high, as well as coarse particle brown mud flat soil and coarse particle grey soil, which can be found on the low ground between the sand dunes on the marine sandy alluvial plain.

Kujukuri beach, where many Sake breweries are located, is a coastal sandy area $60 \mathrm{~km}$ in length and $10 \mathrm{~km}$ in width. The source of the shore sand is a cliff invasion of the Taito cape in the south and Byobu-ga-ura in the north [4]. We considered the soil formation in this area to be influenced by this cliff invasion in addition to the accumulation of blown sand.

We analysed several environmental factors (distance from the sea, soil type, and hardness and collection depth of the preparation water) using a principal component analysis. The analysis roughly divided the environment of Sake breweries into the coastal vicinity, the river plains and the plateau/mountainous region.

These results indicate that for this group of Sake breweries, as the altitude decreases from the plateau/ mountainous region towards the coastline, the hardness of the preparation water changes from soft water to strong hard or hard water, and the soil type changes from gley soil, grey lowland soil, brown forest soil, and andosol to sand dune regosol, coarse particle brown lowland soil, and coarse particle grey soil.

We suggest that the soil type and water quality of Sake breweries in the coastal region (including the former natural coast) have influenced by land areas (the river plains and the plateau/mountainous region), but the extent of this relationship will require further investigation.

\section{ACKNOWLEDGEMENTS}

We wish to express our sincere gratitude to the people from the Sake breweries in Chiba Prefecture, Japan, who cooperated with the survey. This research was supported by JSPS KAKENHI Grant Number 23780019.

\section{REFERENCES}

[1] Kawai, T. and Inoue, M. (2003) Field observation of soil water in sand dune: From the surface to the water table. Soil moisture monitoring in diverse regions. Terrestrial Environment Research Center, University of Tsukuba, Tsukuba, 20-28.

[2] Kaneko, K., Oshida, K. and Mathushima, H. (2012) Ecosystem services on coastal sand beach, the significance as provisioning, regulating, cultural services. Japanese Journal of Landscape Ecology, 17, 19-24. doi:10.5738/jale.17.19

[3] Inoue, M. (2009) Promotion of empirical study to sediment transport system problem from mountainous district to river and sea area. Science and Technology Trend May, 2009, 19-32.

[4] Hirayama, S. (2005) Study on topographical formation in Kujyukuri beach. Master Thesis, University of Tokyo, Tokyo.

[5] Uchiyama, Y. (2001) Tidal circulation of groundwater flow formed in sandy-beach aquifers. Japan Society of Civil Engineers, 670, 37-48.

[6] Brown, A.C. and Mclachlan, A. (1990) Ecology of sandy shores. Elsevier, Amsterdam.

[7] Morikawa, H. (1979) The Landform evolution of the Kujukuri Coastal Plain, central Japan1. The Quaternary Research, 18, 1-16. doi:10.4116/jaqua.18.1

[8] Kaneko, F. (1994) Groundwater quality in Chiba Prefecture characterized with the solution equilibria and the stability diagrams of Minerals. Japanese Journal of Society of Soil Science and Plant Nutrition, 65, 175-183.

[9] Kaneko, F., Imaizumi, M., Hamada, H. and Komae, T. (1994) The aquifer coefficient and the condition of groundwater flow of marine sandy alluvial plain in Chiba Prefecture. Japanese Journal of Society of Soil Science and Plant Nutrition, 65, 297-303.

[10] Food and Bevarege Oeganization (NPO) (2009) Motoi of Japanese Sake-To you who aims at the professional, Japanese Sake sommelier of Japanese Sake, Lecture Text. Sake Service Institute, Tokyo.

[11] Ikawa, R., Shimada, J., Saeki, K. and Taniguchi, M. (2005) Groundwater flow system in Shizuoka plain. Japanese Journal of Geochemistry, 39, 107-118. 